\title{
Rate-equation approach to atomic-laser light statistics.
}

\author{
Laurent Chusseau* \\ Centre d'Électronique et de Micro-optoélectronique de Montpellier, \\ UMR $550^{7}$ CNRS, Université Montpellier II, F34095 Montpellier, France \\ Jacques Arnaud \\ Mas Liron, F30440 Saint Martial, France \\ Fabrice Philippe用 \\ Laboratoire d'Informatique de Robotique et de Microélectronique de Montpellier, \\ UMR 5506 CNRS, 161 Rue Ada, F34392 Montpellier, France
}

(Dated: October 26, 2018)

\begin{abstract}
We consider three- and four-level atomic lasers that are either incoherently (unidirectionally) or coherently (bidirectionally) pumped, the single-mode cavity being resonant with the laser transition. The intra-cavity Fano factor and the photo-current spectral density are evaluated on the basis of rate equations. According to that approach, fluctuations are caused by jumps in active and detecting atoms. The algebra is considerably simpler than the one required by Quantum-Optics treatments. Whenever a comparison can be made, the expressions obtained coincide. The conditions under which the output light exhibits sub-Poissonian statistics are considered in detail. Analytical results, based on linearization, are verified by comparison with Monte Carlo simulations. An essentially exhaustive investigation of sub-Poissonian light generation by three- and four-level atoms lasers has been performed. Only special forms were reported earlier.

PACS numbers: 42.55.Ah, 42.50.Ar, 42.55.Px, 42.50.Lc
\end{abstract}

\section{INTRODUCTION}

Interest in the statistics of light emitted by atomic lasers has been recently revived as a result of the realization of micro-lasers [1]. The main purpose of this paper is to show that expressions derived from rate equations coincide with those previously derived from Quantum Optics. This is so even when the generated light exhibits sub-Poissonian statistics. An introduction to that method as it pertains to sub-Poissonian light generation can be found in tutorial papers [2, 3]. Let us recall that light is called sub-Poissonian when the variance of the number of photo-detection events over some large time duration is less than the average number of events. Equivalently, one may say that the spectral density of the photo-current is less than the shot-noise level at small Fourier (or baseband) frequencies. Analytical expressions are obtained from rate equations in a straightforward manner. Namely, the expression for the internal cavity statistics of many 4-level atoms with a negligible spontaneous decay previously given in Eq. (4) by Ritsch et al. [4] is recovered exactly (see Eq. 20 of the present paper). Similarly, 3-levels atoms expressions obtained by Khazanov et al. [5] are recovered. But coherentlypumped 3-level atoms lasers were apparently not treated

\footnotetext{
*Electronic address: chusseau@univ-montp2.fr; URL: http: //www . opto.univ-montp2.fr/ chusseau

TElectronic address: arnaudj2@wanadoo.fr

$¥$ Also at MIAp, Université Paul Valéry. F34199 Montpellier,

France; Electronic address: Fabrice.Philippe@univ-montp3.fr
}

earlier. If the upper and lower decay times of 4-level atoms tend to zero, the laser is equivalent to a 2-levels atoms laser with Poissonian pump [6]. In that limit, the expressions reported in Arnaud and Estéban [7] in 1990 are recovered. When the above approximations are not applicable, the presently reported expressions appear to be new.

The active medium is a collection of $N$ identical atoms, with the levels labeled $|0\rangle,|1\rangle,|2\rangle$, and $|3\rangle$ in increasing order of energy (see Fig. 1). Level separations are supposed to be large compared with $k_{\mathrm{B}} T$, where $T$ denotes the optical cavity temperature and $k_{\mathrm{B}}$ the Boltzmann constant, so that thermally-induced transitions are negligible. Note that the 3-level atoms schemes in Fig. 11(a) and 1(b) are not special cases of the 4-level scheme in Fig. 11(c). They are treated separately in appendices. Levels $|1\rangle$ and $|2\rangle$ are resonant with the field of a singlemode optical cavity. The active medium is supposed to be strongly homogeneously broadened so that atomic polarizations may be adiabatically eliminated, an approximation applicable also to YAG lasers, $\mathrm{CO}_{2}$ lasers and semiconductor lasers. Such lasers may operate in the steady-state regime, that is, pulsation and chaos do not normally occur.

The probability per unit time that an electronic transition from level $|1\rangle$ to level $|2\rangle$ occurs is taken as equal to $m$, and the probability of an electronic transition from $|2\rangle$ to $|1\rangle$ as $m+1$, where $m$ denotes the number of photons in the cavity (the qualification "per unit time" is henceforth omitted for the sake of brevity). This amounts to selecting a time unit whose typical value depends on the gain medium. Spontaneous decay from level $|2\rangle$ to level 
(a)

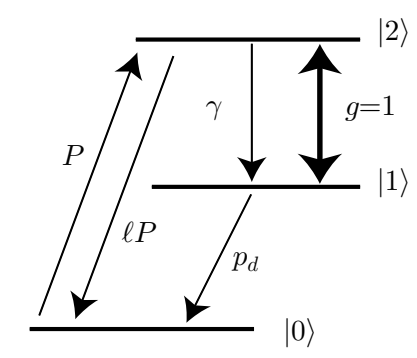

(b)

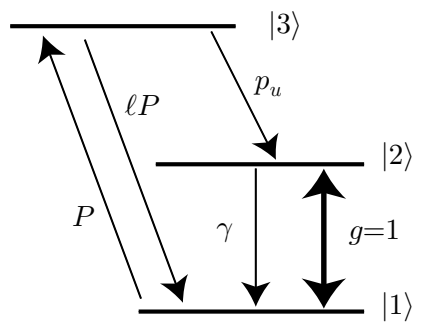

(c)

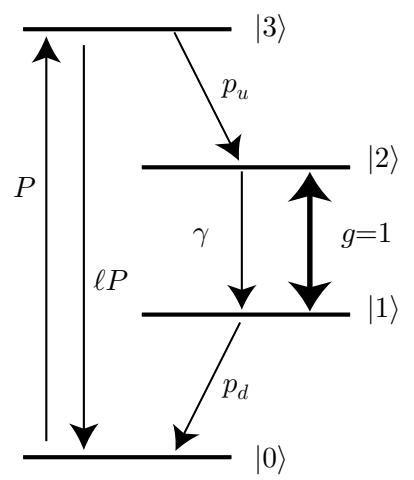

FIG. 1: Level schemes for atomic lasers. (a) $\Lambda$-type 3-level laser, (b) V-type 3-level laser, (c) 4-level atoms laser. For incoherent pumping $\ell=0$. For coherent pumping $\ell=1$.

$|1\rangle$ is allowed with probability $\gamma$. This decay may be either non-radiative or involve radiation into other electromagnetic modes, besides the one of interest. Photons are absorbed with probability $\alpha m$, where $\alpha$ denotes a constant, the absorbing atoms residing most of the time in their ground state. These absorbing atoms model the transmission of light through mirrors with subsequent absorption by a detector. Provided detection is linear and reflectionless, it is immaterial whether absorption occurs inside or outside the optical cavity. For simplicity, internal absorption is neglected.

"Incoherent" pumping promotes electrons from level $|0\rangle$ to level $|3\rangle$ with probability $P$. When transitions from $|0\rangle$ to $|3\rangle$ and from $|3\rangle$ to $|0\rangle$ are both allowed with equal probabilities, the pumping process is called "coherent", following an accepted terminology. We find it convenient to denote by $\ell P$ the $|3\rangle \rightarrow|0\rangle$ transition probability, with $\ell=0$ for incoherent pumping and $\ell=1$ for coherent pumping. Coherent pumping is physically realized by submitting the atoms to strong optical fields nearly resonant with the $|0\rangle \rightarrow|3\rangle$ transition. This pumping field may possibly originate from frequency-filtered thermal radiation and be highly multimode. Levels $|0\rangle$ and $|3\rangle$ need not be sharp. Instead, they may consist of narrow bands for improved coupling to broad-band pumps. One-way incoherent pumping would be appropriate to describe laser-diode pumps. In semiconductors, however, working levels spread into conduction and valence bands, so that the present model would not be adequate. Laser diodes have been treated previously on the basis of rate equations in [3]. Spontaneous decay from level $|3\rangle$ to the upper working level $|2\rangle$ occurs with probability $p_{u}$, and spontaneous decay from the lower working level $|1\rangle$ to the ground level with probability $p_{d}$, where "u" stands for "up" and "d" for down. The relevant probabilities are schematized in Fig. 1 .

One of the best-known laser-noise theory is probably that of Scully and Lamb [8]. Incoherent pumping is modeled by independent injection of 2-level atoms in the optical cavity. This model leads to a photo-count statistics which is, at best, Poissonian. More recently, Ritsch et al. [4], Khazanov et al. [5], Ralph and Savage [9] considered the situation in which the pumping-levels populations may fluctuate. At first, it would seem that this may only increase the noise. It turns out, however, that population fluctuations are correlated in such a way that the output light fluctuations may be sub-Poissonian. It is difficult to pin point a simple intuitive explanation. It has been observed, however, that when lasers are pumped through a cascade of intermediate levels, pumping tends to be regular 10, 11], a situation somewhat similar to laser-diodes high-resistance driving conditions.

Other means of generating sub-Poissonian light have been considered. Golubev and Sokolov 12] were the first in 1984 to point out that lasers with non-fluctuating pumps should emit sub-Poissonian light. This conclusion has been verified experimentally by Machida et al. 113] with the help of laser diodes driven by high-impedance electrical sources. The Scully and Lamb model has been generalized to account for regular atom injection [14, 15. Kolobov et al. [16] made the interesting observation that the photodetection rate spectral density may be below the shot-noise level at non-zero Fourier frequencies in the case of Poissonian pumps. However, the photodetection rate spectral density remains at the shot-noise level at zero frequency. Accordingly, such lasers do not generate sub-Poissonian light in the sense defined earlier. It has been shown that 3-level lasers with coherent decay to the ground state [17, 18] generate sub-Poissonian light, and further that Raman lasers may generate sub-Poissonian light [19]. We will not consider here these more exotic configurations. A review is in [20].

Rate equations treat the number of photons in the cavity as a classical random function of time. The light field is quantized as a result of matter quantization and conservation of energy, but not directly. Rate equations should be distinguished from semi-classical theories in which the optical field is driven by atomic dipole expectation values. The theory employed in this paper rests instead on the consideration of transition probabilities, as 
in the Loudon [21 treatment of optical amplifier noise, for example. Every absorption event reacts on the number of light quanta in the optical cavity. Semi-classical theories are unable to explain sub-Poissonian light statistics because the light generation process and the light detection processes are considered separately. The expressions obtained from rate equations are found to coincide with Quantum Optics results when the number of atoms is large and transitions other than those relating to the atom-cavity interaction are incoherent. The laser is treated as a birth-death Markov process (see Sec. II). A Monte Carlo simulation gives the evolution of the number $m$ of photons in the cavity from which the Fano factor $\mathscr{F}=\operatorname{var}(m) /\langle m\rangle$ is obtained. On the other hand, the instants $t_{k}$ when photons are being absorbed provide the spectral density of the photo-current, whose normalized value $\mathscr{S}$ is unity for Poisson processes. The normalized spectrum is denoted $\mathscr{S}(\Omega)$. The Fourier angular frequency $\Omega$ is called for short: "frequency".

When both the number of atoms and the pumping level increase, the computing time becomes prohibitively large because of the exponentially growing number of events to process. Analytical results are obtained by applying the weak-noise approximation. We first give in Sec. III A the steady-state photon number. The photo-current spectral density at zero frequency is evaluated in Sec. IIIB, and the photo-current spectrum in Sec. III G. The method of evaluation of the intra-cavity Fano factor is in Sec. IIID.

\section{MONTE CARLO METHOD}

The rate-equation model of a $N$-atoms singlemode laser straightforwardly leads to a master equation for the probability of having $m$ photons stored in the cavity at time $t$ [3, 8, 21]. Alternatively, the laser evolution is modeled as a temporally homogeneous birth-death Markov process. In the steady-sate regime, rate of change and equilibrium probabilities of having $m$ and $m+1$ photons within the cavity are linked via a detailed balancing condition. This is a favorable condition for a Monte Carlo simulation 222 because every laser microstate belongs to a Markov chain and will thus occurs proportionally to its equilibrium probability when the number of step increases to $\infty$.

For the $\mathrm{V}$-type lasers considered in the present section (see Fig. 1(b)), rates of change $W_{j}$ are ascribed to the different kinds of events as given in Table 1 . For example, the probability that an atom jumps from level $|1\rangle$ to level $|3\rangle$ during the elementary time interval $[t, t+\delta t]$ is $W_{2} \delta t$, where $\delta t$ is chosen small enough that this probability be much less than unity. Because atoms are coupled to one another only through the field, $W_{2}$ is proportional to the number $n_{1}$ of atoms in $|1\rangle$ at time $t$, and thus $W_{2}=P n_{1}$, where the constant $P$ is proportional to the pump strength. If a jump does occur, $n_{1}$ is reduced by 1 while the number $n_{3}$ of atoms in level $|3\rangle$ is incremented by 1 . If the initial value of $n_{3}$ is $N$ the event
TABLE I: Elementary events in V-type 3-level lasers (see Fig. $1(\mathrm{~b}))$ and corresponding rate of change $W_{j}$.

\begin{tabular}{rcl}
\hline \hline Event & Transition & Rate \\
photon absorption & - & $W_{1}=\alpha m$ \\
pump absorption & $|1\rangle \rightarrow|3\rangle$ & $W_{2}=P n_{1}$ \\
pump emission & $|3\rangle \rightarrow|1\rangle$ & $W_{3}=\ell P n_{3}$ \\
coherent emission & $|2\rangle \rightarrow|1\rangle$ & $W_{4}=(m+1) n_{2}$ \\
coherent absorption & $|1\rangle \rightarrow|2\rangle$ & $W_{5}=m n_{1}$ \\
spontaneous decay & $|2\rangle \rightarrow|1\rangle$ & $W_{6}=\gamma n_{2}$ \\
upper decay & $|3\rangle \rightarrow|2\rangle$ & $W_{7}=n_{3} p_{u}$ \\
\hline \hline
\end{tabular}

does not occur. Similar observations apply to the other jump probabilities. Notice that the coherent emission rate $W_{4}$ is proportional to $m+1$, following the Einstein prescription. This ensures that laser emission re-starts if extinction occurs. A key feature that distinguishes the present formulation from other rate-equation methods is that absorption of photons by the detector is included in the system description. Because detection is supposed to be linear, such events are taken to occur with a rate $W_{1}=\alpha m$, where $\alpha$ expresses detector absorption.

An efficient algorithm has actually been employed [23]: Given that an event of any kind occurred at time $\tau_{k}$, the next-event time is

$$
\tau_{k+1}=\tau_{k}+\frac{1}{\sum_{j} W_{j}} \ln \left(\frac{1}{\mathfrak{r}}\right),
$$

where $\mathfrak{r}$ is a random number uniformly distributed in the interval $[0,1]$. The probability that the event is of kind $l$ is equal to $W_{l} / \sum_{j} W_{j}$. The Monte Carlo method was implemented on a desk computer. Only the total number of atoms in each state needs to be tracked (For fermions the numerical procedure is significantly more involved [24]). Within the whole time set $\left\{\tau_{k}\right\}$, we are mostly interested in the subset $\left\{t_{k}\right\}$ of photon-absorption events. It is straightforward in principle to evaluate the photo-detection noise. We also record $m\left(t_{k}\right)$ to evaluate the mean value and variance of $m$.

The intra-cavity Fano factor $\mathscr{F}$ is represented in Fig. 2. Both coherent (Fig. 2(a)) and incoherent (Fig. 2(b)) pumping are considered. The analytical results in Sec. IIID, based on linearization agree well with the simulation. Notice that $\mathscr{F}$ is below unity within some pumping range. There is good agreement with previous Quantum-Optics results 25].

The normalized spectral density $\mathscr{S}(\Omega)$ is represented in Fig. 3 for incoherent (a) and coherent (b) pumps, and two sets of parameter-values. For each Monte Carlo run, $\mathscr{S}(\Omega)$ is first evaluated from the $\left\{t_{k}\right\}$ list [24 and refined using a smoother power spectral density estimator 26, 27. Averaging over runs and concatenating neighboring frequencies produce the final data together with error bars at the $95 \%$ confidence level. There is fair agreement between Monte Carlo simulations and analytical 
(a)

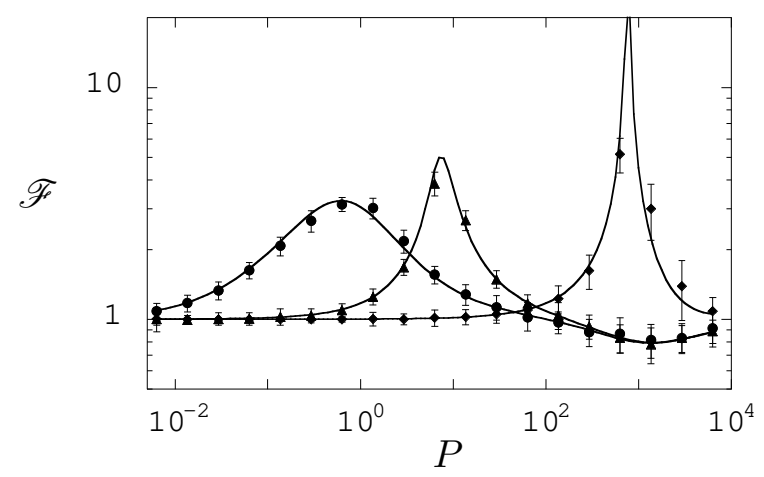

(b)

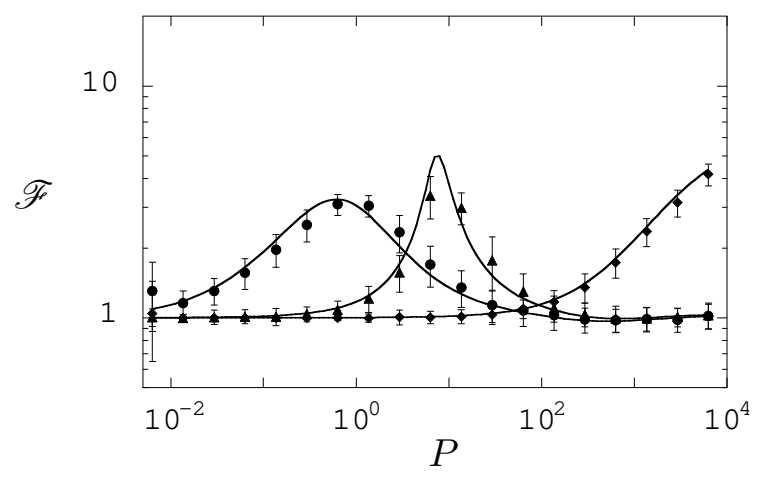

FIG. 2: Intra-cavity Fano factor for V-type lasers as a function of the pumping rate $P$. (a) incoherent pumping, (b) coherent pumping. Error bars are the $95 \%$ confidence level from a statistical treatment applied to ten Monte Carlo runs, each having duration $T_{m}=200$. Plain lines are analytical (see Sec. IIID). The parameters are: $N=100, p_{u}=632$, $\alpha=6.32 . \bullet, \gamma=0 ; \boldsymbol{\Delta}, \gamma=6.32 ; \diamond, \gamma=632$.

formulas to be subsequently reported. Both predict subPoissonian photo-current statistics. Even with one billion photon-absorption events, Monte Carlo spectra exhibit large error bars. An analytical method is to be preferred when it exists. On the other hand, Monte Carlo simulations do not rely on linearization and provide a useful check.

\section{FOUR-LEVELS ATOMS LASERS}

\section{A. Steady-state}

Let $n_{j}, j=0,1,2,3$, denote the number of atoms in state $j$, with

$$
n_{0}+n_{1}+n_{2}+n_{3}=N
$$

The transition rate from $|1\rangle \rightarrow|2\rangle$ (stimulated absorption) is set equal to the number $m$ of photons in the cavity, a rule that defines a time unit. Pumping of an atom from level $|0\rangle$ to level $|3\rangle$ occurs with rate $P$. We allow for a transition rate $\ell P$ that an atom in level $|3\rangle$ decays back to level $|0\rangle$. Atoms in state $|3\rangle$ spontaneously decay (a)

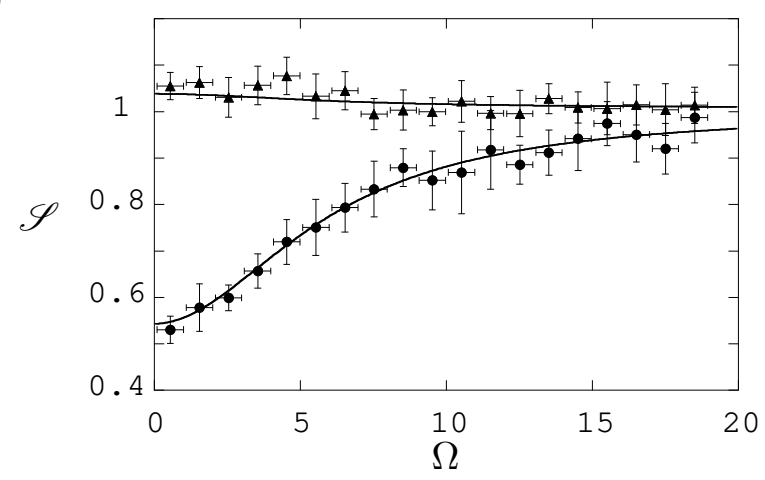

(b)

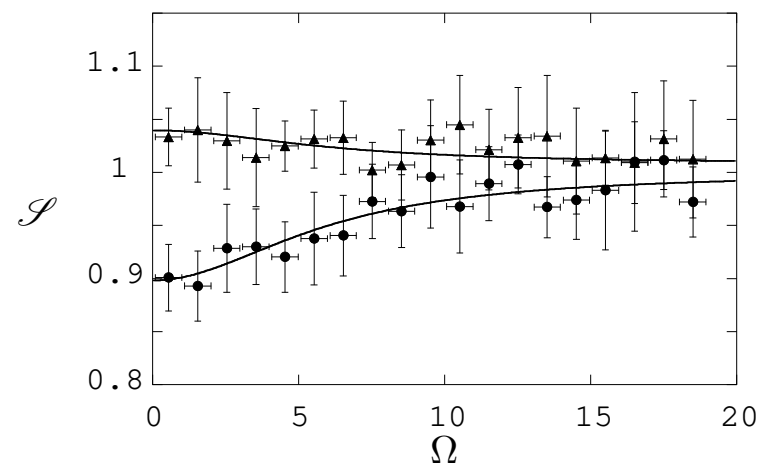

FIG. 3: Normalized photo-current spectral density $\mathscr{S}$ of Vtype lasers as a function of Fourier frequency $\Omega$. Points with error bars are from Monte Carlo simulations with 150 runs, each of duration $T_{m}=100$. Plain lines are analytical (see Sec. III ()). The parameters are: $N=100, p_{u}=632, \alpha=$ 6.32. (a) incoherent pumping $\bullet, \gamma=0$ and $P=1265$; $\boldsymbol{\Lambda}$, $\gamma=6.32$ and $P=20$. (b) coherent pumping, $\bullet, \gamma=0$ and $P=422 ; \boldsymbol{\Lambda}, \gamma=6.32$ and $P=20$.

to state $|2\rangle$ with rate $p_{u}$, while atoms in state $|1\rangle$ spontaneously decays to state $|0\rangle$ with rate $p_{d}$. These spontaneous decays are labelled "upper" and "lower" decay, respectively. Note that when $1 / p_{u}=0$ the population of level $|3\rangle$ vanishes and any dependence on $\ell$ must therefore drop out. Photons are absorbed with rate $\alpha m$. Spontaneous decay from the upper to the lower working levels occurs with rate $\gamma$. Altogether, there are eight kinds of events that may occur in the course of time.

Let $\mathcal{J}$ denotes the net pumping rate, $\mathcal{R}$ the net stimulated rate, $\mathcal{U}$ and $\mathcal{D}$ the upper and lower decay rates, $\mathcal{S}$ the spontaneous decay rate from the upper to the lower working levels, and $\mathcal{Q}$ the photon absorption rate. The steady-state conditions then read

$$
\begin{aligned}
& \mathcal{J}=\mathcal{U}=\mathcal{D}=\mathcal{R}+\mathcal{S}, \\
& \mathcal{Q}=\mathcal{R},
\end{aligned}
$$

where

$$
\begin{array}{ll}
\mathcal{J}=P n_{0}-\ell P n_{3}, & \mathcal{U}=p_{u} n_{3}, \\
\mathcal{R}=(m+1) n_{2}-m n_{1}, & \mathcal{D}=p_{d} n_{1}, \\
\mathcal{S}=\gamma n_{2}, & \mathcal{Q}=\alpha m .
\end{array}
$$


Equations (2), (3) and (何) provide the steady-state atomic populations $n_{i}$ and photon number $m$. In particular

$$
m=\frac{1}{2}\left(\mathscr{B}+\sqrt{\mathscr{B}^{2}+4 \mathscr{P} \mathscr{N}}\right)
$$

where

$$
\begin{aligned}
\mathscr{N} & =\frac{N}{\alpha} \\
\mathscr{P} & =\left[\frac{1}{P}+\frac{2}{p_{d}}+\frac{1+\ell}{p_{u}}\right]^{-1}, \\
\mathscr{B} & =\mathscr{P}\left[\mathscr{N}-1+\frac{1}{p_{d}}(1+\gamma-\mathscr{N} \gamma)\right]-\gamma-1 .
\end{aligned}
$$

For moderate pump powers above threshold, $m$ increases linearly with $P$. The intercept with the $m=0$ axis defines the threshold pump power. Mathematically, this amounts to first replacing in previous equations $m+1$ by $m$, and then setting $m=0$. The laser is found to oscillate for some pump rate provided

$$
\gamma<(\mathscr{N}-1)\left[\frac{\mathscr{N}+1}{p_{d}}+\frac{1+\ell}{p_{u}}\right]^{-1} .
$$

The limiting case is obtained at infinite pump power

$$
\lim _{P \rightarrow \infty} m=\frac{(\mathscr{N}-1)\left(p_{d}-\gamma\right)}{(1+\ell) \frac{p_{d}}{p_{u}}+2}-\gamma
$$

Figure 4 shows how the steady-state photon number evolves as a function of the pumping parameter $P$ for various values of the spontaneous decay rate $\gamma$. Parameters are similar to 25] except $p_{u}$-values chosen here for a perfect superimposition of the coherent and incoherent plots. Moreover it corresponds to optimum pump-noise suppression as derived in Sec. III B. At very high pump levels $m$ saturates like Eq. (8) because of ground-state population depletion.

\section{B. Zero-frequency noise}

Our analytical results rest on a weak-noise approximation. Populations split into steady-state values and fluctuations. For example the instantaneous photon number $m$ is written as $\langle m\rangle+\Delta m$, where $\langle m\rangle$ denotes the steady-state value. Rates split into steady-state values and fluctuations consisting of a deterministic function of the population fluctuations and uncorrelated Langevin "forces". For example $\mathcal{J}$ split into $J=\langle\mathcal{J}\rangle$ and $\Delta J$. The latter is the sum of a deterministic function of the population fluctuations and a Langevin force $j(t)$ expressing the jump process randomness. Thus

$$
\begin{array}{ll}
\mathcal{J} \equiv J+\Delta J, & \mathcal{U} \equiv U+\Delta U, \\
\mathcal{R} \equiv R+\Delta R, & \mathcal{D} \equiv D+\Delta D, \\
\mathcal{S} \equiv S+\Delta S, & \mathcal{Q} \equiv Q+\Delta Q .
\end{array}
$$

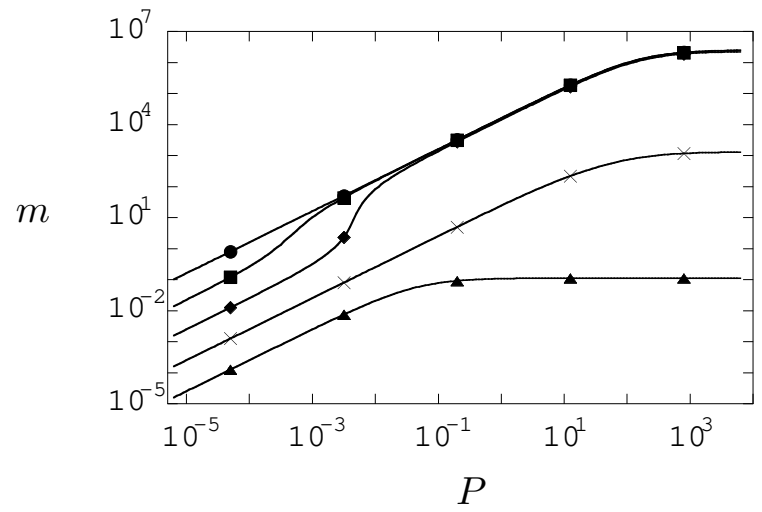

FIG. 4: Steady-state number of photons in 4-level atoms lasers as a function of $P / p_{d}$ for different values of the spontaneous decay rate $\gamma . N=10^{5}, \alpha=6.32, p_{d}=632$. For incoherent pumping $p_{u}=316$. For coherent pumping $p_{u}=949$. $\bullet, \gamma=0 ; \mathbf{\square}, \gamma=6.32 ; \boldsymbol{\vee}, \gamma=63.2 ; \times, \gamma=632 ; \boldsymbol{\Delta}, \gamma=6325$.

where

$$
\begin{aligned}
\Delta J= & P \Delta n_{0}-\ell P \Delta n_{3}+j, \\
\Delta R= & (m+1) \Delta n_{2}-m \Delta n_{1} \\
& +\left(n_{2}-n_{1}\right) \Delta m+r, \\
\Delta S= & \gamma \Delta n_{2}+s, \\
\Delta U= & p_{u} \Delta n_{3}+u, \\
\Delta D= & p_{d} \Delta n_{1}+d, \\
\Delta Q= & \alpha \Delta m+q .
\end{aligned}
$$

A first-order variation of the expressions in Eq. (1) has been performed.

Conservation of the rates gives

$$
\begin{aligned}
& \Delta J=\Delta U=\Delta D=\Delta R+\Delta S, \\
& \Delta Q=\Delta R .
\end{aligned}
$$

Since the total number $N$ of atoms is constant we have

$$
\Delta n_{0}+\Delta n_{1}+\Delta n_{2}+\Delta n_{3}=0 .
$$

Replacing atomic populations and photon numbers by their steady-state values in Sec. III A, the above set of equations can be solved. In particular, $\Delta Q$ is a linear combination of the Langevin forces

$$
\Delta Q=\sum_{z \in\{j, d, u, q, r, s\}} c_{z} z,
$$

where the $c_{z}$ are real coefficients that depend on the parameters $N, P, \ell, p_{u}, p_{d}, \gamma$ and $\alpha$. The detailed expressions, too lengthy to be given here in their general form, are conveniently handled using symbolic calculations.

The normalized zero-frequency photo-current spectral density is of the form

$$
\mathscr{S}=\frac{1}{\alpha m} \sum_{z \in\{j, d, u, r, s\}} c_{z}^{2} \sigma_{z},
$$


where $\sigma_{z}$ denotes the spectral density value of the Langevin noise source $z$, equal to average rates.

$$
\begin{array}{llrl}
\sigma_{j} & =P n_{0}+\ell P n_{3}, & & \sigma_{u}=p_{u} n_{3}, \\
\sigma_{r} & =(m+1) n_{2}+m n_{1}, & & \sigma_{d}=p_{d} n_{1}, \\
\sigma_{s} & =\gamma n_{2}, & \sigma_{q} & =\alpha m .
\end{array}
$$

When these expressions are introduced in Eq. (14) an analytical expression of $\mathscr{S}$ is obtained. Three special cases are considered below, (a) $\gamma=0$ and $m$ large compared with unity, (b) $N \gg \alpha$, (c) $\gamma=0$ and $N \gg \alpha$.

(a) If spontaneous decay is negligible $(\gamma=0)$, Eq. (14) yields

$$
\begin{aligned}
\mathscr{S}=1+\frac{2}{(\mathscr{N}-1)^{2}}+\frac{8 \mathscr{P}^{2}}{p_{d}^{2}}+\frac{(6-4 \mathscr{N}) \mathscr{P}}{(\mathscr{N}-1) p_{d}} \\
+\frac{2(1+\ell) \mathscr{P}^{2}}{p_{u}^{2}}+\frac{2 \mathscr{P}\left(2 \mathscr{P}-p_{d}\right)}{p_{d} p_{u}},
\end{aligned}
$$

where $\mathscr{P}$ and $\mathscr{N}$ are defined in Eq. (6). The normalized spectral density is unity at low and high pumping levels. For some constant $\mathscr{N}$ value, $\mathscr{S}$ reaches its minimum valued

$$
\mathscr{S}_{\text {min }}=\frac{2 \mathscr{N}(\mathscr{N}-1)+11+\ell(4 \mathscr{N}(\mathscr{N}-1)+15)}{2(3+4 \ell)(\mathscr{N}-1)^{2}},
$$

when

$$
\frac{P}{p_{u}}=\frac{1}{1+\ell}, \quad \frac{P}{p_{d}}=\frac{\mathscr{N}(1+2 \ell)-(2+3 \ell)}{(1+\ell)(2 \mathscr{N}-1)} .
$$

(b) When $N \gg \alpha$, Eq. (14) yields

$$
\begin{gathered}
\mathscr{S}=1+\frac{2 \gamma}{p_{d}-\gamma}-\frac{4 \mathscr{P}+2 \gamma}{p_{d}}+\frac{8 \mathscr{P}(\mathscr{P}+\gamma)}{p_{d}^{2}}-\frac{8 \mathscr{P}^{2} \gamma}{p_{d}^{3}} \\
+\frac{2 \mathscr{P}\left(\gamma-p_{d}\right)\left(p_{d}-2 \mathscr{P}\right)}{p_{d}^{2} p_{u}}-\frac{2(1+\ell) \mathscr{P}^{2}\left(\gamma-p_{d}\right)}{p_{d} p_{u}^{2}} .
\end{gathered}
$$

Figure 5 gives the normalized zero-frequency photocurrent spectral density $\mathscr{S}\left(P / p_{d}, \gamma / p_{d}\right)$ in the form of contour plots, selecting $P / p_{u}=2 P / p_{d}$ for the case of incoherent pumping and $P / p_{u}=\frac{2}{3} P / p_{d}$ for the case of coherent pumping. The darker the area, the lower is the spectral density. Since dark areas are wider in Fig. 5 (a) than in Fig. F(b), incoherent pumping is to be preferred. Figure 5 shows that sub-Poissonian light generation by optically pumped 4-level atom lasers is robust against spontaneous decay and pumping conditions. The optimum conditions (darkest areas) are defined in Eq. (18). But small departures from these conditions do not increase much the noise.

(c) If both $\gamma=0$ and $N \gg \alpha$, the spectral density obtained either by setting $\mathscr{N}=\infty$ in Eq. (16) or $\gamma=0$ in Eq. (19), reads

$$
\mathscr{S}=1-\frac{2 P p_{d} p_{u}\left(p_{d}+2\left(P+P \ell+p_{u}\right)\right)}{\left(2 P p_{u}+p_{d}\left(P+P \ell+p_{u}\right)\right)^{2}}
$$

TABLE II: Minimum value of the zero-frequency photocurrent spectral density $\mathscr{S}_{\text {min }}$ and intra-cavity Fano factor $\mathscr{F}$ for 3 and 4 -levels atoms lasers. The conditions on $P, p_{u}$ and $p_{d}$ are given. Spontaneous decay from the upper working level is neglected and it is assumed that $N \gg \alpha$.

\begin{tabular}{c|c|c|c}
\hline \hline Laser & $\mathscr{S}_{\text {min }}$ & $\mathscr{F}$ & Conditions \\
\hline$\Lambda$-type 3-level & $1 / 2$ & $3 / 4$ & $p_{d}=2 P$ \\
$\Lambda$-type 3-level & \\
& $2 / 3$ & $5 / 6$ & $p_{d}=3 P$ \\
V-type 3-level $^{a}$ & $1 / 2$ & $3 / 4$ & $p_{u}=\frac{1}{2} P$ \\
V-type 3-level $^{b}$ & $5 / 6$ & $11 / 12$ & $p_{u}=\frac{3}{2} P$ \\
$4-$ level $^{a}$ & $1 / 3$ & $2 / 3$ & $p_{u}=P, p_{d}=2 P$ \\
4 -level & $3 / 7$ & $5 / 7$ & $p_{u}=2 P, p_{d}=\frac{4}{3} P$ \\
\hline \hline
\end{tabular}

${ }^{a}$ Incoherent pumping.

${ }^{b}$ Coherent pumping.

an expression that coincides with Eq. (4) of [4]. The absolute minimum value and corresponding conditions are obtained from Eqs. (17) and (18)

$$
\mathscr{S}_{\min }=\frac{1+2 \ell}{3+4 \ell}, \quad \frac{P}{p_{u}}=\frac{1}{1+\ell}, \quad \frac{P}{p_{d}}=\frac{1+2 \ell}{2(1+\ell)} .
$$

For incoherent pumping, $\ell=0$, we have therefore $\mathscr{S}_{\text {min }}=1 / 3$ when $p_{u}=P$ and $p_{d}=2 P$. For coherent pumping, $\ell=1$, we have $\mathscr{S}_{\text {min }}=3 / 7$ when $p_{u}=2 P$ and $p_{d}=\frac{4}{3} P$.

Table III gives the minimum spectral density values achievable with optically pumped three- and 4-level lasers as obtained from Eqs. (21), (B6) and (A6). Under the very special condition of negligible spontaneous decay and $N \gg \alpha$, the intra-cavity Fano factor depends linearly of the zero-frequency normalized photo-current spectral density [12], $\mathscr{S}=2 \mathscr{F}-1$. Such relation does not hold in the general situation where Fano factors follow from the formulation in Sec. IIID.

V-type incoherently-pumped lasers were treated earlier by Khazanov et al. [5]. Ralph and Savage [9, 28] extended the analysis to incoherently pumped $\Lambda$-type lasers and 4-level atom lasers. Ritsch et al. [1 gave a description of 4-level lasers for the two pumping schemes. These previous results are exactly recovered from the present rate-equations method.

Formulas derived in the present paper for coherentlypumped 3-level lasers, however, appear to be new. Because the present method involves simple algebra the conditions under which the photo-current spectral density is minimum are easily obtained. To our knowledge, all the results presented in this paper when $\gamma$ does not vanish, or $N$ is not much larger than $\alpha$, are new.

\section{Photo-detection spectrum}

The steady-state relations were given in Eq. (11). At some Fourier frequency $\Omega$ the generalized rate equations 
(a)

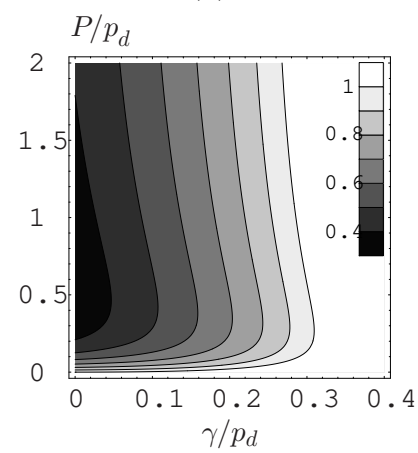

(b)

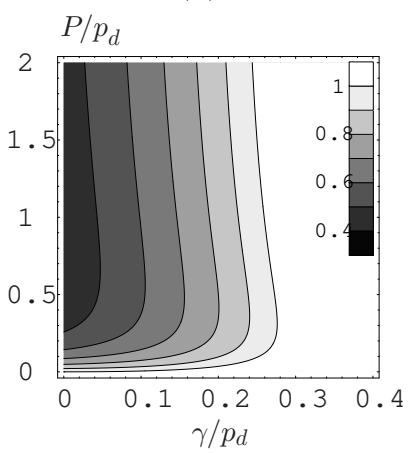

FIG. 5: Contour plots of the zero-frequency normalized photo-current spectral density $\mathscr{S}$ for 4 -level atoms lasers as a function of $\gamma / p_{d}$ and $P / p_{d}$. The laser parameters are the same as in Fig. 4, namely: $N=10^{5}, \alpha=6.32, p_{d}=632$. (a) incoherent pumping, $p_{u}=316$, (b) coherent pumping, $p_{u}=949$. In the white area the light statistics is super-Poissonian.

read [2]

$$
\begin{aligned}
& i \Omega \Delta m=\Delta R-\Delta Q, \\
& i \Omega \Delta n_{0}=\Delta D-\Delta J, \\
& i \Omega \Delta n_{1}=\Delta R+\Delta S-\Delta D, \\
& i \Omega \Delta n_{2}=\Delta U-\Delta R-\Delta S, \\
& i \Omega \Delta n_{3}=\Delta J-\Delta U,
\end{aligned}
$$

Similar to Sec. III B, equations Eqs. (9), (10), (12) and (22) are solved for $\Delta Q$. The formula for the light spectral density $\mathscr{S}$ is the same as Eq. (14) except that the coefficients $\tilde{c}_{z}$ are complex and frequency dependent

$$
\mathscr{S}(\Omega)=\frac{1}{\alpha m} \sum_{z \in\{j, d, u, r, s\}} \tilde{c}_{z}(\Omega) \tilde{c}_{z}^{\star}(\Omega) \sigma_{z},
$$

where the Langevin "forces" $\sigma_{z}$ are still given in Eq. 15.

After rearranging, Eq. (23) gives the spectral density in the form

$$
\mathscr{S}(\Omega)=1+\frac{a_{3} \Omega^{6}+a_{2} \Omega^{4}+a_{1} \Omega^{2}+a_{0}}{\Omega^{8}+b_{3} \Omega^{6}+b_{2} \Omega^{4}+b_{1} \Omega^{2}+b_{0}},
$$

where the coefficients $a_{i}$ and $b_{i}$ are real. The form in Eq. (24) ensures that $\mathscr{S}(\Omega)$ tends to unity (shot-noise level) at high frequencies.

Figure 6 shows that $\mathscr{S}$ reaches its minimum value at $\Omega=0$. When spontaneous decay from the upper working level may be neglected, light is always sub-Poissonian and the lowest $\mathscr{S}$-value occurs when $P / p_{d}=1 / 2$. Spontaneous decay from the upper working level is inconsequential until $\gamma / p_{d} \approx 310^{-2}$. The light statistics ceases to be sub-Poissonian when $P / p_{d}>0.3$.

\section{Fano factor}

The intra-cavity photon statistics is characterized by the Fano factor $\mathscr{F}=\left\langle\Delta m^{2}\right\rangle /\langle m\rangle$, where $m$ denotes as

(a)

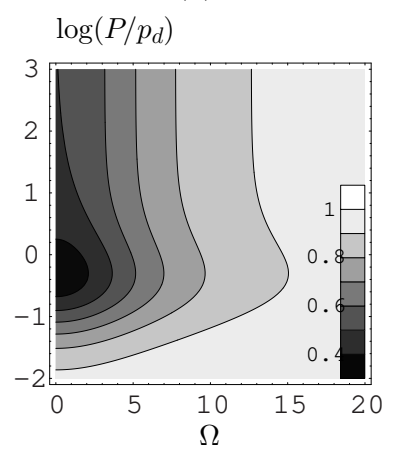

(b)

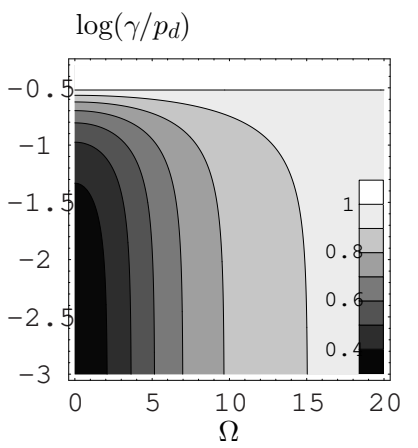

FIG. 6: Contour plots of normalized photo-current spectra $\mathscr{S}(\Omega)$ for incoherently pumped 4 -level atoms lasers. The laser parameters are the same as in Fig. 4, namely: $N=10^{5}$, $\alpha=6.32, p_{d}=632, p_{u}=316$. (a) dependence of $\mathscr{S}(\Omega)$ on $P / p_{d}$ with $\gamma=0$. (b) dependence of $\mathscr{S}(\Omega)$ on $\gamma / p_{d}$ with $P=316$.

before the number of photons in the cavity. The variance of $m$ equals the integration over frequency of $\mathscr{S}_{\Delta m}(\Omega)$, the spectral density of $\Delta m$. Similarly to Sec. III G, $\mathscr{S}_{\Delta m}(\Omega)$ obtains by solving for $\Delta m$ instead of $\Delta Q$ and its general form is

$$
\mathscr{S}_{\Delta m}(\Omega)=\frac{a_{3}^{\prime} \Omega^{6}+a_{2}^{\prime} \Omega^{4}+a_{1}^{\prime} \Omega^{2}+a_{0}^{\prime}}{\Omega^{8}+b_{3}^{\prime} \Omega^{6}+b_{2}^{\prime} \Omega^{4}+b_{1}^{\prime} \Omega^{2}+b_{0}^{\prime}},
$$

where $a_{i}^{\prime}$ and $b_{i}^{\prime}$ are real coefficients. The Fano factor is thus

$$
\mathscr{F}=\int_{-\infty}^{\infty} \mathscr{S}_{\Delta m}(\Omega) \frac{\mathrm{d} \Omega}{2 \pi}
$$

The Fano factor for incoherently pumped 4-level atoms lasers is represented as a function of pump and spontaneous decay rates in Fig. 6 .

At small pumping rates, and when the spontaneous decay rate $\gamma$ is large, the photon statistic is essentially that of amplified thermal light and the Fano factor $\mathscr{F}=$ $\langle m\rangle+1$. The inset in Fig. 7 shows that this simple result holds indeed for $\gamma=632$, excepts for the highest values of $P$, where a slight reduction of $\mathscr{F}$ occurs.

When $\gamma<p_{d}$, the Fano factor exhibits a peak just below threshold [29], and decreases to $\approx 1$ until the ground level gets significantly depleted. Self-quenching [25, 30] is not observed here because atomic polarizations have been adiabatically eliminated. Otherwise, our results fully agree with those reported by Koganov and Shuker [25].

\section{CONCLUSION}

We have considered optically-pumped 4-level and 3level atom lasers in resonant single-mode cavities. The light statistics has been obtained from a simple rateequation approach, using both a Monte Carlo simulation 


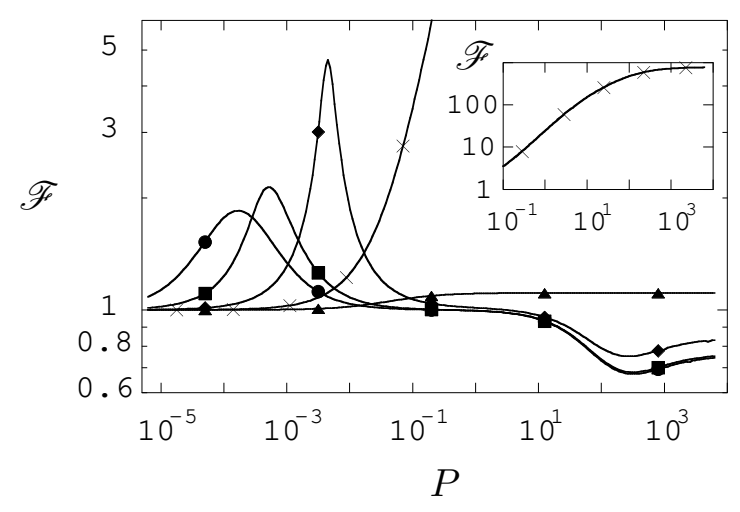

FIG. 7: Internal Fano factor of incoherently pumped 4-level atoms lasers as a function of $P$ and $\gamma ; \bullet, \gamma=0 ; \mathbf{\square}, \gamma=6.32$; $\diamond, \gamma=63.2 ; \times, \gamma=632 ; \boldsymbol{\Delta}, \gamma=6325$. The inset is for $\gamma=632$. The laser parameters are the same as in Fig. 4 . namely: $N=10^{5}, \alpha=6.32, p_{d}=632$.

and an analytical method based on linearization. The emitted light may be sub-Poissonian, as was previously observed by many authors. Whenever a comparison can be made, exact agreement is reached with the previous Quantum Optics results. In the case of coherentlypumped 3-level atoms lasers our results appear to be new. Note that 3-level atoms lasers are not special cases of the 4-level scheme. When the assumptions of negligible spontaneous decay and large atom numbers are not made, the results presented in this paper for the internal and external field statistics appear to be new.

For practical reasons, Monte Carlo simulations were restricted to $N \approx 1000$ atoms. Because the analytical formulas, obtained through the use of symbolic calculus, are lengthy they were not written down in the paper. However, they were employed to determine the conditions under which the spectral density of the photo-current reaches its minimum value. For example, we found that when spontaneous decay from the upper working level may be neglected, 3-level atoms lasers may deliver light with fluctuations at half the shot-noise level. 4-level atoms lasers may deliver light with fluctuations at one third of the shot-noise level. The photo-current noise decreases further and tends to zero, under ideal conditions, when the number of levels becomes large [11].

\section{APPENDIX A: $\Lambda$-TYPE LASERS}

The number $m$ of photons in the steady-state is still

$$
m=\frac{1}{2}\left(\mathscr{B}+\sqrt{\mathscr{B}^{2}+4 \mathscr{P} \mathscr{N}}\right)
$$

with

$$
\begin{aligned}
\mathscr{N}= & \frac{N}{\alpha}, \\
\mathscr{P}= & {\left[\frac{1}{P}+\frac{2+\ell}{p_{d}}\right]^{-1}, } \\
\mathscr{B}= & \mathscr{P}\left[\mathscr{N}-1-\ell+\frac{1}{p_{d}}((1+\ell)(1+\gamma)-\mathscr{N} \gamma)\right] \\
& -1-\gamma .
\end{aligned}
$$

Lasing may occur if

$$
\gamma<\frac{\mathscr{N}-1-\ell}{\mathscr{N}+1} p_{d}
$$

(a) When spontaneous decay may be neglected, $\gamma=$ 0 , the zero-frequency normalized photo-current spectral density reads

$$
\begin{aligned}
\mathscr{S}=1+\frac{2(1+\ell+\ell \mathscr{N})}{(1+\ell-\mathscr{N})^{2}} & +\frac{4(2+\ell) \mathscr{P}^{2}}{p_{d}^{2}} \\
& -\frac{2(3+2 \ell-2 \mathscr{N}) \mathscr{P}}{(1+\ell-\mathscr{N}) p_{d}} .
\end{aligned}
$$

Note that when $\ell=0$ this expression may be obtained by setting $1 / p_{u}=0$ in the 4 -level expression. This is not, however, a valid procedure in general.

(b) When $N \gg \alpha$

$$
\begin{aligned}
& \mathscr{S}=1+\frac{2 \gamma}{p_{d}-\gamma}-\frac{2(2 \mathscr{P}+\gamma)}{p_{d}} \\
& +\frac{2 \mathscr{P}(2(2+\ell) \mathscr{P}+(4+\ell) \gamma)}{p_{d}^{2}}-\frac{4(2+\ell) \mathscr{P}^{2} \gamma}{p_{d}^{3}} .
\end{aligned}
$$

(c) When $\gamma=0$ and $N \gg \alpha$

$$
\mathscr{S}=1-\frac{4 P p_{d}}{\left(P(2+\ell)+p_{d}\right)^{2}}
$$

The minimum value of $\mathscr{S}$ and corresponding value of $P$ are

$$
\mathscr{S}_{\text {min }}=\frac{1+\ell}{2+\ell}, \quad P=\frac{p_{d}}{2+\ell} .
$$

\section{APPENDIX B: V-TYPE LASERS}

The number $m$ of photons in the steady-state is still

$$
m=\frac{1}{2}\left(\mathscr{B}+\sqrt{\mathscr{B}^{2}+4 \mathscr{P} \mathscr{N}}\right),
$$

with

$$
\begin{aligned}
\mathscr{N}= & \frac{N}{2 \alpha} \\
\mathscr{P}= & {\left[\frac{1}{P}+\frac{1+2 \ell}{2 p_{u}}\right]^{-1}, } \\
\mathscr{B}= & \frac{\mathscr{P}}{4 p_{u}}\left[(2 \mathscr{N}-1)\left(\gamma+2 p_{u}\right)-1\right] \\
& -\frac{1}{2}-\frac{\gamma}{2}-\mathscr{N} \gamma .
\end{aligned}
$$


Lasing may occur if

$$
\gamma<\frac{2 \mathscr{N}-1}{1+\ell+2 \ell \mathscr{N}} p_{u}
$$

(a) When spontaneous decay may be neglected, $\gamma=$ 0 , the zero-frequency normalized photo-current spectral density reads

$$
\begin{aligned}
\mathscr{S}=1+\frac{2 \mathscr{N}+1}{(2 \mathscr{N}-1)^{2}}+\frac{(1+2 \ell) \mathscr{P}^{2}}{2 p_{u}^{2}} & \\
& -\frac{(4 \mathscr{N}-1) \mathscr{P}}{2(2 \mathscr{N}-1) p_{u}} .
\end{aligned}
$$

(b) When $N \gg \alpha$

$$
\begin{aligned}
\mathscr{S} & =1+\frac{2 \gamma}{\mathscr{P}-\gamma}-\frac{\mathscr{P}}{p_{u}}+\frac{\mathscr{P}(\mathscr{P}(1+2 \ell)-\gamma)}{2 p_{u}^{2}} \\
& +\frac{(1+2 \ell) \mathscr{P}^{2} \gamma}{4 p_{u}^{3}}-\frac{2 \mathscr{P}^{2} \gamma}{(\mathscr{P}-\gamma)\left(\mathscr{P} \gamma+2(\mathscr{P}-\gamma) p_{u}\right)} .
\end{aligned}
$$

(c) When $\gamma=0$ and $N \gg \alpha$

$$
\mathscr{S}=1-\frac{4 P p_{u}}{\left(P(1+2 \ell)+2 p_{u}\right)^{2}}
$$

The minimum value of $\mathscr{S}$ and corresponding value of $P$ are

$$
\mathscr{S}_{\text {min }}=\frac{1+4 \ell}{2+4 \ell}, \quad P=\frac{2 p_{u}}{1+2 \ell} .
$$

\section{ACKNOWLEDGMENTS}

This work was supported by the STISS Department of Université Montpellier II and by CNRS under the JemSTIC Program.
[1] K. An, J. J. Childs, R. R. Dasari, and M. S. Feld, Phys. Rev. Lett. 73, 3375 (1994).

[2] J. Arnaud, Opt. Quantum Electron. 27, 63 (1995).

[3] J. Arnaud, Opt. Quantum Electron. (2001), to appear, quant-ph/0201151.

[4] H. Ritsch, P. Zoller, C. W. Gardiner, and D. F. Walls, Phys. Rev. A 44, 3361 (1991).

[5] A. M. Khazanov, G. A. Koganov, and E. P. Gordov, Phys. Rev. A 42, 3065 (1990).

[6] L. Chusseau, J. Arnaud, and F. Philippe, unpublished.

[7] J. Arnaud and M. Estéban, IEE Proc. J 137, 55 (1990).

[8] M. O. Scully and W. E. Lamb, Jr., Phys. Rev. 159, 208 (1967).

[9] T. C. Ralph and C. M. Savage, Phys. Rev. A 44, 7809 (1991).

[10] H. Ritsch and P. Zoller, Phys. Rev. A 45, 1881 (1992).

[11] H.-J. Briegel, G. M. Meyer, and B.-G. Englert, Phys. Rev. A 53, 1143 (1996).

[12] Y. M. Golubev and I. V. Sokolov, Sov. Phys.-JETP 60, 234 (1984).

[13] S. Machida, Y. Yamamoto, and Y. Itaya, Phys. Rev. Lett. 58, 1000 (1987).

[14] R. B. Levien, M. J. Collett, and D. F. Walls, Phys. Rev. A 47, 5030 (1993).

[15] C. Zhu, Phys. Rev. A 48, 3930 (1993).

[16] M. I. Kolobov, L. Davidovich, E. Giacobino, and C. Fabre, Phys. Rev. A 47, 1431 (1993).

[17] F. Haake, M. I. Kolobov, C. Fabre, E. Giacobino, and
S. Reynaud, Phys. Rev. Lett. 71, 995 (1993).

[18] Y. M. Golubev, Theor. Math. Phys+ 109, 1437 (1996).

[19] H. Ritsch, M. A. M. Marte, and P. Zoller, Europhys. Lett. 19, 7 (1992).

[20] L. Davidovitch, Rev. Mod. Phys. 68, 127 (1996).

[21] R. Loudon, The Quantum Theory of Light (Oxford University Press, Oxford, 1983).

[22] D. P. Landau and K. Binder, A Guide to Monte Carlo Simulations in Statistical Physics (Cambridge University Press, Cambridge, 2000).

[23] D. T. Gillespie, Markov Processes: An Introduction for Physical Scientists (Academic Press, San Diego, 1992).

[24] L. Chusseau and J. Arnaud, Opt. Quantum Electron. (2001), to appear, quant-ph/0105078.

[25] G. A. Koganov and R. Shuker, Phys. Rev. A 63, 015802 (2000).

[26] A. Papoulis, Probability, Random Variables, and Stochastic Processes (MacGraw-Hill, New York, 1991).

[27] W. H. Press, S. A. Teukolsky, W. T. Vetterling, and B. P. Flannery, Numerical Recipes in $C$ (Cambridge University Press, Cambridge, 1992).

[28] T. C. Ralph and C. M. Savage, Quantum Opt. 5, 113 (1993).

[29] B. Jones, S. Ghose, J. P. Clemens, and P. R. Rice, Phys. Rev. A 60, 3267 (1999)

[30] Y. Mu and C. M. Savage, Phys. Rev. A 46, 5944 (1992). 